\title{
Relationship between parathyroid mass and parathyroid hormone level in hemodialysis patients with secondary hyperparathyroidism
}

\author{
Li Fang ${ }^{1}$, Bing Tang ${ }^{1}$, Dawei Hou², Meijuan Meng ${ }^{1}$, Mingxia Xiong ${ }^{1}$ and Junwei Yang ${ }^{1 *}$
}

\begin{abstract}
Background: To evaluate the influence of parathyroid mass on the regulation of parathyroid hormone (PTH) secretion, we investigated the relationship between the resected parathyroid gland in total parathyroidectomy and the parathyroid hormone level in hemodialysis patients with secondary hyperparathyroidism.

Methods: From January 2009 to July 2014, 223 patients undergoing total parathyroidectomy were included. The size and the weight of parathyroid gland were measured during the operation.

Results: 874 parathyroid glands were removed. A positive correlation was identified between the size and the weight of resected parathyroid glands. We found that both the preoperative PTH and the reduction of PTH were significantly correlated with the size and the weight of parathyroid glands in a positive manner. However, in the subgroup of patients with PTH $<1000 \mathrm{pg} / \mathrm{ml}$, no significant correlation was found.

Conclusions: Larger parathyroid gland secretes more PTH and high level of serum PTH usually indicated that surgical removal might be required. However, since PTH levels could be influenced by the pharmaceutical drug, the large size of parathyroid gland might be used as a much more appropriate guide that indicates the requirement of surgery treatment even when the parathyroid hormone was less than $1000 \mathrm{pg} / \mathrm{ml}$.
\end{abstract}

Keywords: Secondary hyperparathyroidism, Total parathyroidectomy, Parathyroid gland mass, Parathyroid hormone

\section{Background}

Secondary hyperparathyroidism characterized by increased secretion of $\mathrm{PTH}$, is one of the major serious complications of patients with chronic renal failure on long-term hemodialysis [1]. In the course of secondary hyperparathyroidism, parathyroid growth progresses gradually from diffuse hyperplasia to nodular hyperplasia, and eventually to formation of adenomas at advanced stages [2-4]. Several clinical studies have documented that although PTH levels can be suppressed by continuous treatment with phosphate binders, vitamin D analogs or calcimimetics [5-7], the progressively reducing expression of calcium-sensing receptors $(\mathrm{CaSR})$ and vitamin $\mathrm{D}$ receptors (VDR) would

\footnotetext{
* Correspondence: jwyang@njmu.edu.cn

${ }^{1}$ Center for Kidney Disease, 2nd Affiliated Hospital, Nanjing Medical University, 262 Zhongshan North Road, Nanjing, Jiangsu Province, China Full list of author information is available at the end of the article
}

result in resistance to treatment $[8,9]$, and, finally, requiring parathyroidectomy (PTX) [10, 11]. Parathyroidectomy was reported to become gradually necessary [12].

In the preoperative assessment of parathyroidectomy, ultrasonography, scintigraphy, and positron emission tomography scans are used to estimate the glandular size and help plan the operative approach. However, imaging studies are usually heavily operator-dependent, which could even explain the wide variability in the reported sensitivity (ranging from 30-80 \%) for the detection of abnormal parathyroid glands [13]. Thus, the surgeons could not be entirely dependent on the imaging techniques. Since the surgical exploration for parathyroid glands and the surgical experience were crucial, successful parathyroidectomy requires an understanding of both the anatomy and the embryology of the parathyroid glands. The surgeons need to learn more about the 
relationship between the parathyroid glands and the parathyroid hormone level. McCarron et al. have reported that PTH induced by ethylene diamine tetraacetic acid (EDTA) response was related to their total gland size measured at the time of their PTX [14]. However, some studies suggested that the altered quality of the parathyroid mass and not only the increased parathyroid mass might be responsible for non-controllable hyperparathyroidism in uremia [9]. The relationship between parathyroid gland mass and the parathyroid hormone levels remains uncertain. In this study, to evaluate the influence of parathyroid mass on the regulation of parathyroid hormone secretion, we investigated the relationship between the parathyroid gland resected in total parathyroidectomy and the parathyroid hormone levels in hemodialysis patients with secondary hyperparathyroidism.

\section{Patients and methods}

\section{Selection of patients}

We performed a retrospective analysis of a prospectively collected cohort of consecutive adult patients who underwent parathyroidectomy (PTX) treatment between January 2009 and July 2014 in our institution. From January 2009 to July 2014, 223 patients undergoing maintenance hemodialysis therapy with biochemical or clinical evidence, or both, of secondary hyperparathyroidism were referred to the second affiliated hospital of Nanjing medical university for parathyroidectomy. Inclusion criteria were as follows: 1) patients receiving dialysis for more than 6 months; 2) serum intact PTH level $>500 \mathrm{pg} / \mathrm{mL}$ on two or more occasions; and/or 3) patients with parathyroid nodular or diffuse hyperplasia identified by ultrasound imaging or radioisotope scan; 4) patients with symptomatic secondary hyperparathyroidism, such as bone and joint pain, pathologic fractures, severe pruritus, restless legs syndrome and so on. In all patients, $4 \mathrm{~h}$ hemodialysis was performed three times a week with bicarbonate dialysate containing a $3.0 \mathrm{mEq} / \mathrm{l}$ calcium concentration. Exclusion criteria included a history of parathyroidectomy, an unstable medical condition during the previous 30 days and the cases with ectopic or residual parathyroid glands.

\section{Study design}

The study protocol was approved by the Ethics Committee of Nanjing Medical University. All patients gave their consent to undergo PTX and participated in this study. The written informed consent was obtained and documented in the legal medical record. In the preoperative preparation, all cases had routine preoperative tests including complete blood counts, biochemical tests, serum electrolytes, coagulation screening, chest $x$-ray, electrocardiogram, heart Doppler ultrasound and so on. The localization of parathyroid glands was evaluated by using neck ultrasonography and parathyroid scintigraphy (termed
Tc-99 m 2 methoxy-isobutyl-isonitrile, 99mTc-MIBI). Despite all this, identification of the exact location is still challenging. During the surgery, exploration for the hyperplasia parathyroid glands was performed with the patient under general anaesthesia, using a transverse collar incision. All patients underwent total parathyroidectomy without autotransplantation. All resected parathyroid glands were measured, weighed and verified histologically. The volume of each parathyroid gland (PTG) was estimated by using the formula: $a * b * c * \pi / 6 \mathrm{~mm} 3$ (where $a, b$, and $\mathrm{c}$ are the dimensions of gland in millimeters).

\section{Analysis of laboratory values}

Blood routine tests were performed using an LH-750 Hematology Analyzer (Beckman Coulter, Inc., Fullerton, CA). Biochemical indices, such as serum albumin, calcium (Ca), phosphorus $(\mathrm{P})$, and alkaline phosphatase (ALP), were measured using an Automatic Biochemical Analyzer (HITACHI 7080; Hitachi, Ltd, Tokyo, Japan). Serum intact-parathyroid hormone (iPTH) levels were measured using a UniCel DxI800 Access Immunoassay System (Beckman Coulter, Inc., Fullerton, CA).

\section{Statistical analyzes}

Statistical analysis was done with SPSS $15 \mathrm{~J}$ for Windows (SPSS, Chicago, IL, USA) to observe any significant differences. All data is shown as the mean \pm S.D., unless otherwise indicated. The relationship between the intact PTH levels and the weight, the volume, the maximum diameter of parathyroid glands were investigated by Spearman's rank correlation coefficient. P-values $<0.001$ were considered significant.

\section{Results}

Clinical parameters of hemodialysis (HD) patients undergoing parathyroidectomy

As shown in Table 1, the patients' ages ranged from 16 to 70 years (average, $47.6 \pm 11.11$ years); They had undergone maintenance hemodialysis therapy for $1 \sim 26$ years (average, $8.3 \pm 3.64$ years). 126 patients were men and 97 were women. The frequency distribution can be seen in Fig. 1.

Based on the criteria suggested by Gagne et al. [15], surgical procedure was considered successful in all patients whose serum levels of iPTH dropped from mean values of $1556.9 \pm 879.27 \mathrm{pg} / \mathrm{ml}$ to $16.0 \pm 44.76 \mathrm{pg} / \mathrm{ml}$ (ANOVA, $P<0.001$ ). More than $90 \%$ decline in 24 h-postoperative PTH reliably predicts operative success.

Correlation of the weight with the size of parathyroid gland In 223 patients, 874 glands were resected (Table 2). 214 patients had 4 parathyroid glands. Three glands were removed in 8 patients. As shown in Fig. 2c, the sizes of parathyroid gland were measured. By histopathology, all patients had the diagnosis of nodular parathyroid hyperplasia. After operation, pruritus, bone pain and muscle weakness disappeared. 
Table 1 Patient characteristics

\begin{tabular}{lll}
\hline Characteristic & Patients $(\mathrm{n}=223)$ & Normal range \\
\hline Age, years & $47.6 \pm 11.11$ & - \\
Gender (male/female) & $126 / 97$ & - \\
Dialysis vintage, years & $8.3 \pm 3.64$ & - \\
Kt/N & $1.4 \pm 0.19$ & $1.2 \sim 1.8$ \\
Calcium,mmol/L & $2.51 \pm 0.23$ & $2.15 \sim 2.55 \mathrm{mmol} / \mathrm{L}$ \\
Phosphorus, mmol/L & $2.11 \pm 0.52$ & $0.81 \sim 1.45 \mathrm{mmol} / \mathrm{L}$ \\
albumin-corrected calcium, mmol/L & $2.47 \pm 0.22$ & - \\
Albumin, g/dl & $41.8 \pm 4.37$ & $39.7 \sim 49.4 \mathrm{~g} / \mathrm{L}$ \\
calcium-phosphorus product & $64.9 \pm 17.50$ & - \\
Alkaline phosphatase (U/l) & $405.5 \pm 423.97$ & $40 \sim 129 \mathrm{U} / \mathrm{L}$ \\
Intact PTH, pg/ml (preoperative) & $1556.9 \pm 879.27$ & $12 \sim 88 \mathrm{pg} / \mathrm{ml}$ \\
Intact PTH, pg/ml (postoperative $1 \mathrm{st}$ day) & $16.0 \pm 44.76$ & $12 \sim 88 \mathrm{pg} / \mathrm{ml}$ \\
\hline
\end{tabular}

Data are expressed as mean \pm S.D

We calculated the total weight and volume of all resected glands for each patient. As shown in Fig. 2a and $\mathrm{b}$, a positive correlation was identified between the mass and the weight of resected parathyroid glands $(P<0.001$, $R=0.967$ for the volume; $P<0.001, R=0.847$ for the maximum diameter;).
Correlation of the preoperative intact-PTH and the reduction of intact-PTH with the size of parathyroid gland

Figures $3 \mathrm{a}, \mathrm{c}$ and e show the correlation of serum intact PTH with the volume, the weight, and the maximum diameter of parathyroid gland $(P<0.001, R=0.515 ; P<0.001$, $R=0.511 ; P<0.001, R=0.437$; respectively). A positive

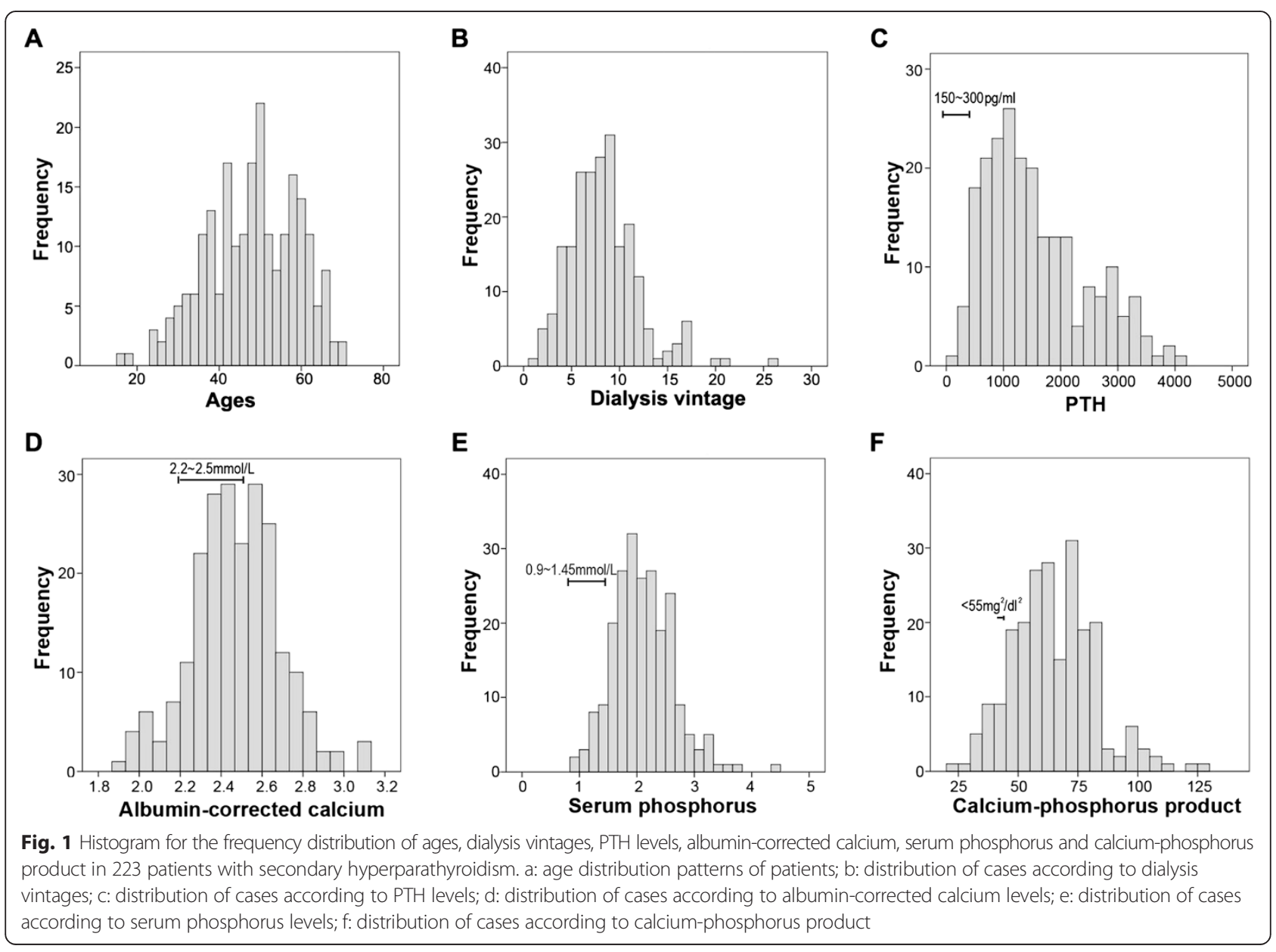


Table 2874 parathyroid glands identified at the primary operation in 223 patients for secondary hyperparathyroidism

\begin{tabular}{lclll}
\hline Location & No. of parathyroid glands & The size $(\mathrm{cm})$ & The volume $\left(\mathrm{cm}^{3}\right)$ & The weight $(\mathrm{g})$ \\
\hline Right superior parathyroid gland & 214 & $1.4 \pm 0.57(0.5-3.5)$ & $0.35 \pm 0.69(0.01-4.24)$ & $0.40 \pm 0.65(0.01-3.84)$ \\
Right inferior parathyroid gland & 221 & $1.6 \pm 0.48(0.6-2.8)$ & $0.60 \pm 0.68(0.06-3.27)$ & $0.63 \pm 0.69(0.03-3.7)$ \\
Left superior parathyroid gland & 221 & $1.5 \pm 0.50(0.4-3)$ & $0.38 \pm 0.60(0.02-4.71)$ & $0.40 \pm 0.62(0.01-4.55)$ \\
Left inferior parathyroid gland & 218 & $1.6 \pm 0.51(0.5-3)$ & $0.59 \pm 0.81(0.03-4.70)$ & $0.67 \pm 0.73(0.04-4.32)$ \\
\hline
\end{tabular}

Data are expressed as mean \pm S.D

correlation was also observed between the reduction of intact PTH after surgery and the volume, the weight, and the maximum diameter of parathyroid gland $(P<0.001$, $R=0.513 ; P<0.001, R=0.509 ; P<0.001, R=0.436$; respectively). Therefore, the increased parathyroid mass seems to secrete more PTH.

Then, we divided the patients into 3 subgroups: PTH $<500 \mathrm{pg} / \mathrm{ml}, 500 \sim 1000 \mathrm{pg} / \mathrm{ml}$ and PTH $>1000 \mathrm{pg} / \mathrm{ml}$. In the subgroups of patients with $\mathrm{PTH}<500 \mathrm{pg} / \mathrm{ml}$ and $\mathrm{PTH}<1000 \mathrm{pg} / \mathrm{ml}$, no significant correlation was found between PTH levels and parathyroid gland (Table 3). Whereas, in the subgroups of patients with PTH $>1000 \mathrm{pg} / \mathrm{ml}$, serum intact PTH was correlated significantly in a positive manner with the parathyroid gland mass $(P<0.001, R=0.338 ; P<0.001, R=0.396 ; P<0.001$, $R=0.404$; respectively).

\section{Discussion}

Chronic kidney disease-mineral and bone disorder (CKD-MBD) is a growing health care concern associated

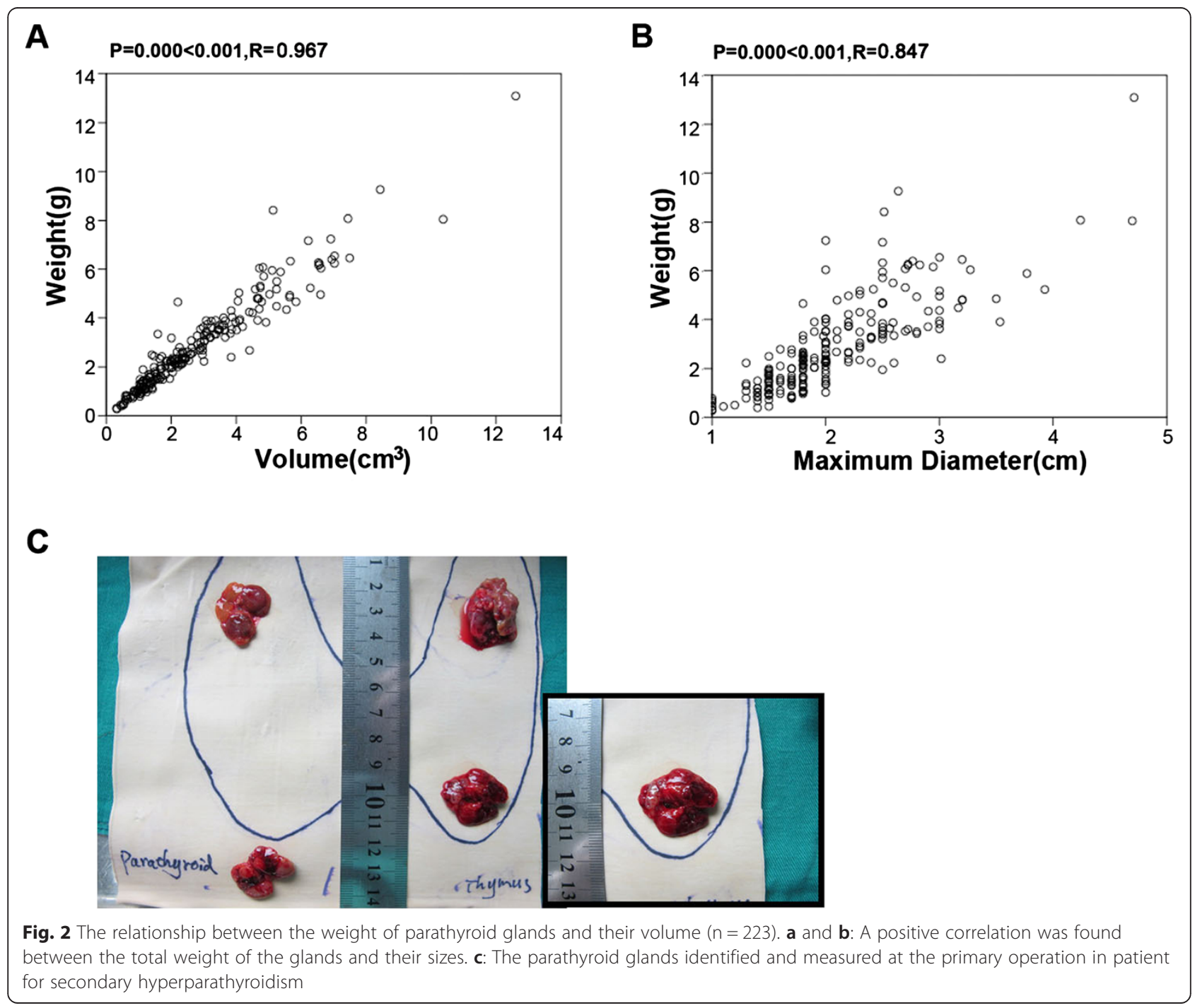




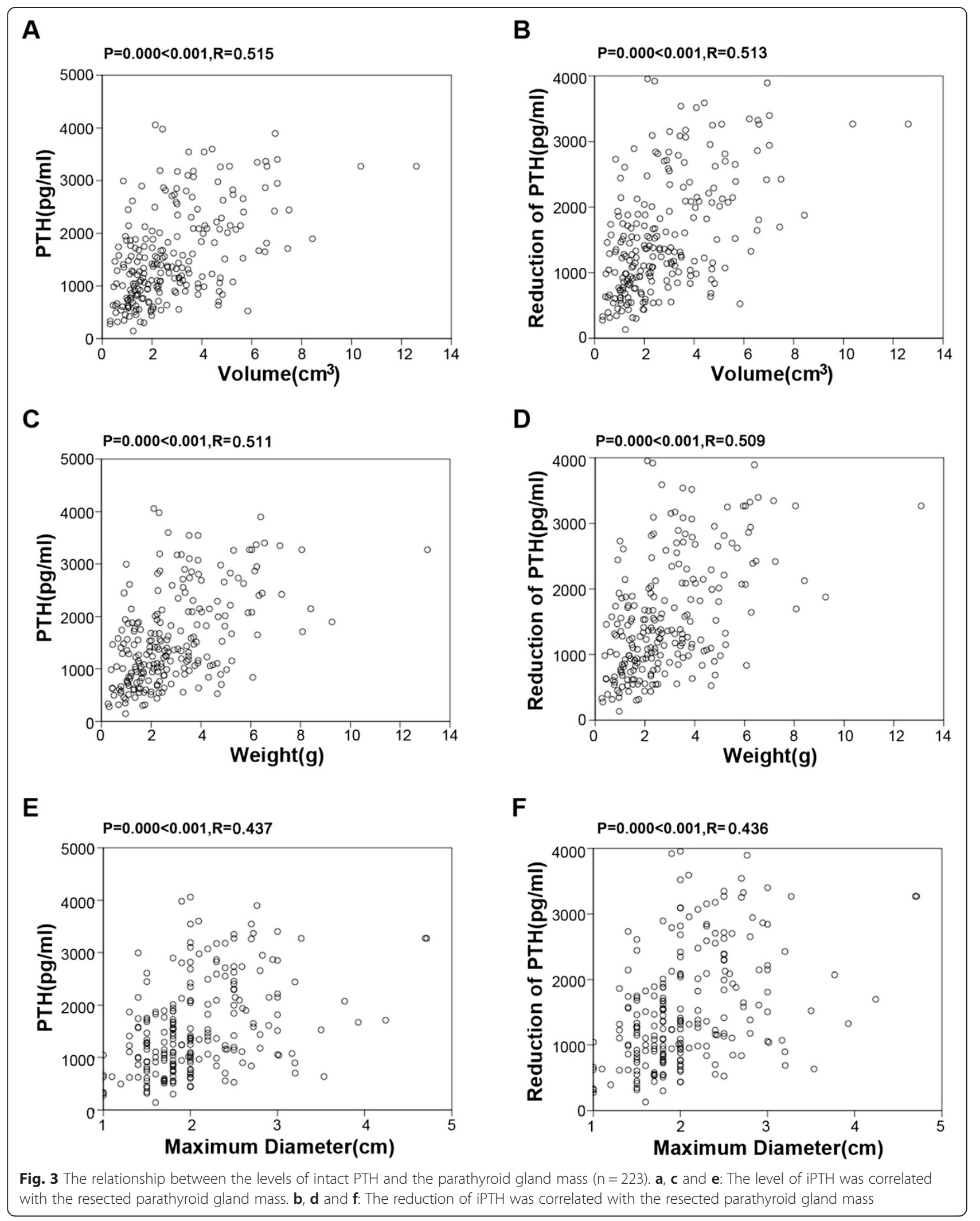


Table 3 Bivariate Pearson's correlation analysis in three subgroups of hemodialysis patients with different serum PTH levels

\begin{tabular}{|c|c|c|c|c|c|c|}
\hline \multirow{2}{*}{$\begin{array}{l}\text { PTH level } \\
(\mathrm{pg} / \mathrm{ml})\end{array}$} & \multicolumn{2}{|c|}{ PTG Volume $\left(\mathrm{cm}^{3}\right)$} & \multicolumn{2}{|c|}{ PTG Weight (g) } & \multicolumn{2}{|c|}{ PTG Maximum Diameter (cm) } \\
\hline & $P$ value & Spearman's rho & $P$ value & Spearman's rho & $P$ value & Spearman's rho \\
\hline$<500$ & 0.592 & 0.172 & 0.957 & 0.018 & 0.948 & 0.021 \\
\hline $500 \sim 1000$ & 0.297 & 0.140 & 0.186 & 0.178 & 0.248 & 0.156 \\
\hline$>1000$ & $0.000^{*}$ & 0.338 & $0.000^{*}$ & 0.396 & $0.000^{*}$ & 0.404 \\
\hline
\end{tabular}

${ }^{*} \mathrm{P}<0.001$, statistically significant

with secondary hyperparathyroidism, mineral abnormalities, and increased risk of cardiovascular disease [16, 17]. As one of the most common abnormalities of CKD-MBD, secondary hyperparathyroidism leads to nodular transformation of the parathyroids in up to $75 \%$ of cases $[18,19]$. Can the increased glandular mass be reduced? Such a reduction would call for massive apoptosis to take place in the parathyroids. However, there are no known stimuli for apoptosis in the parathyroid cells [9]. Thus, poor efficacy of pharmaceutical drug in progressive secondary hyperparathyroidism (SHPT) argues in favour of parathyroidectomy [20-22]. The problem is particularly severe in China. That's because vitamin D analogs and calcimimetics were unavailable in mainland China during the study period. The most common drugs currently used to treat secondary hyperparathyroidism in China were calcium-based phosphorus binders and non-selective VDR activator medications which may cause recurrent hypercalcemia and hyperphosphataemia.

Since the sensitivity of imaging procedures has been reported to be lower in secondary hyperparathyroidism than in primary hyperparathyroidism [23], even with the use of combined neck ultrasonography and parathyroid scintigraphy, bilateral surgical exploration with identification of all glands is required. What's more, since the relatively small size of missed adenomatous glands could always lead to a high rate of re-operation in whom major anaesthesia risks are frequent, the surgeons need to get a general preoperative assessment not only about the localization but also the influence of parathyroid mass on PTH secretion.

The relationship with the parathyroid gland and the parathyroid hormone level was still uncertain until now. Inaba et al. reported that serum bio-PTH but not intact PTH correlated significantly in a positive manner with the maximal diameter of the parathyroid gland [23]. Takatoshi et al. also demonstrated that the secretion of PTH solely depends on the size of the parathyroid glands [24]. However, some evidence hints that the parathyroid mass is to a large extent of less importance for PTH secretion than the 'quality' of parathyroid mass. The 'content' of the different noduli in severe parathyroid hyperplasia is determining the PTH secretion [9]. Since the mass of the parathyroid gland was usually determined by ultrasonography in those studies, the ultrasonography tests are usually heavily operator-dependent and show a wide variability. In our study, we evaluated the mass by directly measuring the three dimensions of the resected parathyroid glands in operation. We found that nodular transformation of the parathyroids was up to $90 \%$ in our cases. Furthermore, a significantly positive correlation between the resected parathyroid gland and the intact serum PTH was found. This data suggested that the larger parathyroid gland might secrete more intact $\mathrm{PTH}$, confirming that the size of a gland could be used as an indication for surgical treatment. However, in the subgroup patients with $\mathrm{iPTH}<1000 \mathrm{pg} / \mathrm{ml}$, no significant correlation was found. The difference might lie in the previous treatment with phosphate binders, vitamin D analogs or calcimimetics. However, large evidence confirmed that PTH levels might return to pre-treatment values when the treatment is stopped [24-26]. What's more, with the condition progress, medicine resistance could be a result of the reducing expression of calcium-sensing receptors (CaSR) and vitamin D receptors (VDR). Thus, we cannot help wondering should we still use repeated nonselective VDR activator in poorly controlled secondary hyperparathyroidism. Our results suggested that PTH levels might be influenced by the pharmaceutical drug under manageable condition, whereas the size of parathyroid gland is to a large extent of more importance on the regulation of PTH secretion. Therefore, the large size of parathyroid gland might be used as a much more appropriate guide that indicates the requirement of surgery treatment even when the parathyroid hormone was less than $1000 \mathrm{pg} / \mathrm{ml}$.

\section{Conclusion}

In summary, nodular transformation of the parathyroids was common in the patients with severe secondary hyperparathyroidism. Generally, larger parathyroid gland secretes more PTH and high level of serum PTH usually indicated that surgical removal might be required. However, since PTH levels could easily be influenced by the pharmaceutical drug, the large size of parathyroid gland might be used as a much more appropriate guide that indicates the requirement of surgery treatment.

\section{Abbreviations}

PTH: Parathyroid hormone; CaSR: Calcium-sensing receptors; VDR: Vitamin D receptors; PTX: Parathyroidectomy; EDTA: Ethylene diamine tetraacetic acid; 
99mTc-MIBI: Tc-99 m methoxy-isobutyl-isonitrile; PTG: Parathyroid gland; Ca: Calcium; P: Phosphorus; ALP: Alkaline phosphatase; iPTH: Intact-parathyroid hormone; CKD-MBD: Chronic kidney disease-mineral and bone disorder; SHPT: Secondary hyperparathyroidism.

\section{Competing interests}

The authors declare that they have no competing interests.

\section{Authors' contributions}

LF participated in the design of the study, collected data, made the figures and table, analyzed and interpreted the data, drafted the manuscript. BT, DH and MX performed the experiments, and collected data; MM helped to perform the statistical analysis. JY conceived the study, drafted the manuscript, and participated in coordination. All authors have given final approval of the version to be published.

\section{Acknowledgement}

This work was supported by the Key Project of Science and Technology Bureau of Jiangsu Province (No. BL2013037) to Junwei Yang.

\section{Author details}

${ }^{1}$ Center for Kidney Disease, 2nd Affiliated Hospital, Nanjing Medical University, 262 Zhongshan North Road, Nanjing, Jiangsu Province, China. 'Department of General Surgery, 2nd Affiliated Hospital, Nanjing Medical University, 262 Zhongshan North Road, Nanjing, Jiangsu Province, China.

Received: 29 September 2014 Accepted: 21 May 2015

Published online: 10 June 2015

\section{References}

1. Guideline Working Group, Japanese Society for Dialysis Therapy. Clinical practice guideline for the management of secondary hyperparathyroidism in chronic dialysis patients. Ther Apher Dial. 2008, 12(6):514-525.

2. Drueke TB. Cell biology of parathyroid gland hyperplasia in chronic renal failure. J Am Soc Nephrol. 2000;11(6):1141-52.

3. Rodriguez M, Canalejo A, Garfia B, Aguilera E, Almaden Y. Pathogenesis of refractory secondary hyperparathyroidism. Kidney Int Suppl. 2002:80:155-60.

4. Cozzolino M, Brancaccio D, Gallieni M, Galassi A, Slatopolsky E, Dusso A. Pathogenesis of parathyroid hyperplasia in renal failure. J Nephrol. 2005;18(1):5-8

5. Coburn JW, Maung HM, Elangovan L, Germain MJ, Lindberg JS, Sprague SM, et al. Doxercalciferol safely suppresses PTH levels in patients with secondary hyperparathyroidism associated with chronic kidney disease stages 3 and 4 . Am J Kidney Dis. 2004;43(5):877-90

6. Block GA, Martin KJ, de Francisco AL, Turner SA, Avram MM, Suranyi MG, et al. Cinacalcet for secondary hyperparathyroidism in patients receiving hemodialysis. N Engl J Med. 2004;350(15):1516-25.

7. Chertow GM, Lu ZI, Xu X, Knight TG, Goodman WG, Bushinsky DA, et al. Self-reported symptoms in patients on hemodialysis with moderate to severe secondary hyperparathyroidism receiving combined therapy with cinacalcet and low-dose vitamin D sterols. Hemodial Int. 2012;16(2):188-97.

8. Lewin E, Garfia B, Recio FL, Rodriguez M, Olgaard K. Persistent downregulation of calcium-sensing receptor mRNA in rat parathyroids when severe secondary hyperparathyroidism is reversed by an isogenic kidney transplantation. J Am Soc Nephrol. 2002;13(8):2110-6.

9. Lewin $\mathrm{E}$, Olgaard $\mathrm{K}$. Influence of parathyroid mass on the regulation of PTH secretion. Kidney Int Suppl. 2006;102:S16-21.

10. Fukagawa M, Komaba H, Onishi Y, Fukuhara S, Akizawa T, Kurokawa K. Mineral metabolism management in hemodialysis patients with secondary hyperparathyroidism in Japan: baseline data from the MBD-5D. Am J Nephrol. 2011;33(5):427-37.

11. Yuan CM, Nee R, Narayan R, Abbott KC. Treatment of secondary hyperparathyroidism with parathyroidectomy instead of cinacalcet: time to pick the low-hanging fruit? Am J Kidney Dis. 2012;60(2):179-81.

12. Slinin Y, Foley RN, Collins AJ. Clinical epidemiology of parathyroidectomy in hemodialysis patients: the USRDS waves 1, 3, and 4 study. Hemodial Int 2007;11(1):62-71

13. Fuster D, Ybarra J, Ortin J, Torregrosa JV, Gilabert R, Setoain X, et al. Role of pre-operative imaging using $99 \mathrm{mTc}-\mathrm{MIBI}$ and neck ultrasound in patients with secondary hyperparathyroidism who are candidates for subtotal parathyroidectomy. Eur J Nucl Med Mol Imaging. 2006;33(4):467-73.
14. McCarron DA, Muther RS, Lenfesty B, Bennett WM. Parathyroid function in persistent hyperparathyroidism: relationship to gland size. Kidney Int. 1982;22(6):662-70.

15. Gagne ER, Urena P, Leite-Silva S, Zingraff J, Chevalier A, Sarfati E, et al. Shortand long-term efficacy of total parathyroidectomy with immediate autografting compared with subtotal parathyroidectomy in hemodialysis patients. J Am Soc Nephrol. 1992;3(4):1008-17.

16. Yokoyama K, Taniguchi M, Fukagawa M. A Japanese approach for CKD-MBD. Kidney Int Suppl (2011). 2013;3(5):451-6.

17. Goodman WG, Quarles LD. Development and progression of secondary hyperparathyroidism in chronic kidney disease: lessons from molecular genetics. Kidney Int. 2008;74(3):276-88.

18. Neyer U, Hoerandner H, Haid A, Zimmermann G, Niederle B. Total parathyroidectomy with autotransplantation in renal hyperparathyroidism: low recurrence after intra-operative tissue selection. Nephrol Dial Transplant. 2002;17(4):625-9.

19. Cunningham J, Locatelli F, Rodriguez M. Secondary hyperparathyroidism: pathogenesis, disease progression, and therapeutic options. Clin J Am Soc Nephrol. 2011;6(4):913-21.

20. Komaba H, Nakanishi S, Fujimori A, Tanaka M, Shin J, Shibuya K, et al. Cinacalcet effectively reduces parathyroid hormone secretion and gland volume regardless of pretreatment gland size in patients with secondary hyperparathyroidism. Clin J Am Soc Nephrol. 2010;5(12):2305-14.

21. Naranda J, Ekart R, Pecovnik-Balon B. Total parathyroidectomy with forearm autotransplantation as the treatment of choice for secondary hyperparathyroidism. J Int Med Res. 2011;39(3):978-87.

22. Ichii M, Ishimura E, Okuno S, Chou H, Kato Y, Tsuboniwa N, et al. Decreases in parathyroid gland volume after cinacalcet treatment in hemodialysis patients with secondary hyperparathyroidism. Nephron Clin Pract. 2010;115(3):c195-202.

23. Alexandrides TK, Kouloubi K, Vagenakis AG, Yarmenitis S, Spyridonidis T, Vassilakos $P$, et al. The value of scintigraphy and ultrasonography in the preoperative localization of parathyroid glands in patients with primary hyperparathyroidism and concomitant thyroid disease. Hormones (Athens) 2006;5(1):42-51.

24. Chertow GM, Dillon M, Burke SK, Steg M, Bleyer AJ, Garrett BN, et al. A randomized trial of sevelamer hydrochloride (RenaGel) with and without supplemental calcium. Strategies for the control of hyperphosphatemia and hyperparathyroidism in hemodialysis patients. Clin Nephrol. 1999;51(1):18-26.

25. Slatopolsky EA, Burke SK, Dillon MA. RenaGel, a nonabsorbed calcium- and aluminum-free phosphate binder, lowers serum phosphorus and parathyroid hormone. The RenaGel Study Group. Kidney Int. 1999;55(1):299-307.

26. Akiba T, Marumo F, Owada A, Kurihara S, Inoue A, Chida Y, et al. Controlled trial of falecalcitriol versus alfacalcidol in suppression of parathyroid hormone in hemodialysis patients with secondary hyperparathyroidism. Am J Kidney Dis. 1998;32(2):238-46.

\section{Submit your next manuscript to BioMed Central and take full advantage of:}

- Convenient online submission

- Thorough peer review

- No space constraints or color figure charges

- Immediate publication on acceptance

- Inclusion in PubMed, CAS, Scopus and Google Scholar

- Research which is freely available for redistribution 